\title{
Severe Myasthenic Manifestation of Leptospirosis Associated with New Sequence Type of Leptospira interrogans
}

\section{Matthias Tomschik, ${ }^{1}$ Inga Koneczny, ${ }^{1}$ Anna-Margarita Schötta, Sebastian Scharer, Merima Smajlhodzic, \\ Paloma Fernandes Rosenegger, Martin Blüthner, Romana Höftberger, Fritz Zimprich, Gerold Stanek, Mateusz Markowicz}

We report the rapid development of a myasthenic crisis as the first-time manifestation of myasthenia gravis. The symptoms developed in the course of acute leptospirosis associated with a new sequence type of Leptospira interrogans. Antibiotic treatment led to rapid amelioration of myasthenia.

$\mathrm{L}$ eptospirosis is a global zoonotic disease, endemic in tropical areas, and caused by spirochetes of the genus Leptospira (1). Involvement of the nervous systems is rare, and only single cases involving aseptic meningitis, encephalitis, movement disorders, neuritis, and polymyositis, among others $(2,3)$, have been reported. We report a case of leptospirosis leading to a myasthenic crisis and subsequent diagnosis of a rare form of myasthenia gravis (MG) in a previously healthy traveler returning to Austria from Southeast Asia.

In August 2017, a previously healthy man, 32 years of age, visited an emergency department in Vienna, Austria, because of generalized weakness, malaise, double vision, and a 2-day history of fever and diarrhea. His medical history was unremarkable, and he was not taking any medication. Three days before the onset of symptoms, he had returned from a 4-week vacation in Vietnam and Thailand, where he swam in a waterfall pool and came into contact with indigenous animals. In particular, he helped to wash elephants in northern Thailand 1 week before his return. He reported feeling well upon arrival in Austria and even went white-water rafting in an alpine area the next day without experiencing weakness or malaise. However, 1 day later

Author affiliation: Medical University of Vienna, Vienna, Austria (M. Tomschik, I. Koneczny, A.-M. Schötta, S. Scharer, M. Smajlhodzic, P.F. Rosenegger, R. Höftberger, F. Zimprich, G. Stanek, M. Markowicz); Medizinisches Versorgungszentrum Labor PD Dr. Volkmann und Kollegen GbR, Karlsruhe, Germany (M. Blüthner)

DOI: https://doi.org/10.3201/eid2505.181591 he noticed fatigability in his legs when walking up stairs, and nonbloody diarrhea developed. The diarrhea subsided, but other symptoms progressively worsened until he had trouble swallowing and walking.

A physical examination at the emergency department revealed elevated temperature $\left(38.0^{\circ} \mathrm{C}\right)$, pulse $(138 \mathrm{bpm})$, and blood pressure $(146 / 104 \mathrm{~mm} \mathrm{Hg})$. The patient displayed typical myasthenic symptoms, including bilateral ptosis, Cogan's lid twitch signs, bilateral weakness of ocular movements, and dysarthria. Symptoms worsened with prolonged talking, including dysphagia and myasthenic weakness of all limbs, such that the patient was dependent on a wheelchair and unable to raise his arms.

Laboratory diagnostic tests revealed elevated C-reactive protein $(27.72 \mathrm{mg} / \mathrm{dL}$ [reference $<0.50 \mathrm{mg} / \mathrm{dL}$ ] $)$ and gamma-glutamyl-transferase (491 U/L [reference $<60$ $\mathrm{U} / \mathrm{L}]$ ), with left shift of leukocytes and proteinuria. Other laboratory parameters were within reference limits. In cerebrospinal fluid, cell counts and protein concentration were within reference limits, but intrathecal IgG production was evident.

Real-time PCR on EDTA blood targeting the gene for the major outer membrane protein lip L32 of human pathogenic Leptospira spp. showed positive results. We then subjected the sample strain to multilocus sequence typing (MLST) (4) to investigate its relationship with other L. interrogans strains and determine whether the infection was acquired during the patient's stay in Southeast Asia. In MLST analysis, we sequenced and analyzed 7 housekeeping genes of Leptospira spp. ( $g \operatorname{lm} U$, pntA, sucA, tpiA, pfkB, mreA, and caiB). Among these, we found 2 new alleles (for $m r e A$ and tpiA), which resulted in a new sequence type (ST), 247. We constructed a phylogenetic tree for all 147 currently available $L$. interrogans STs, including ST247 (Figure 1) (5). The analysis showed that the isolate we obtained clusters with strains from Asia; however, the MLST database comprises strains predominantly from Asia. Further MLST analysis using eBURST (http://eburst.mlst.net) showed that linking STs were still missing. Further research in this field is required before a convincing phylogeographic conclusion can be reached. 


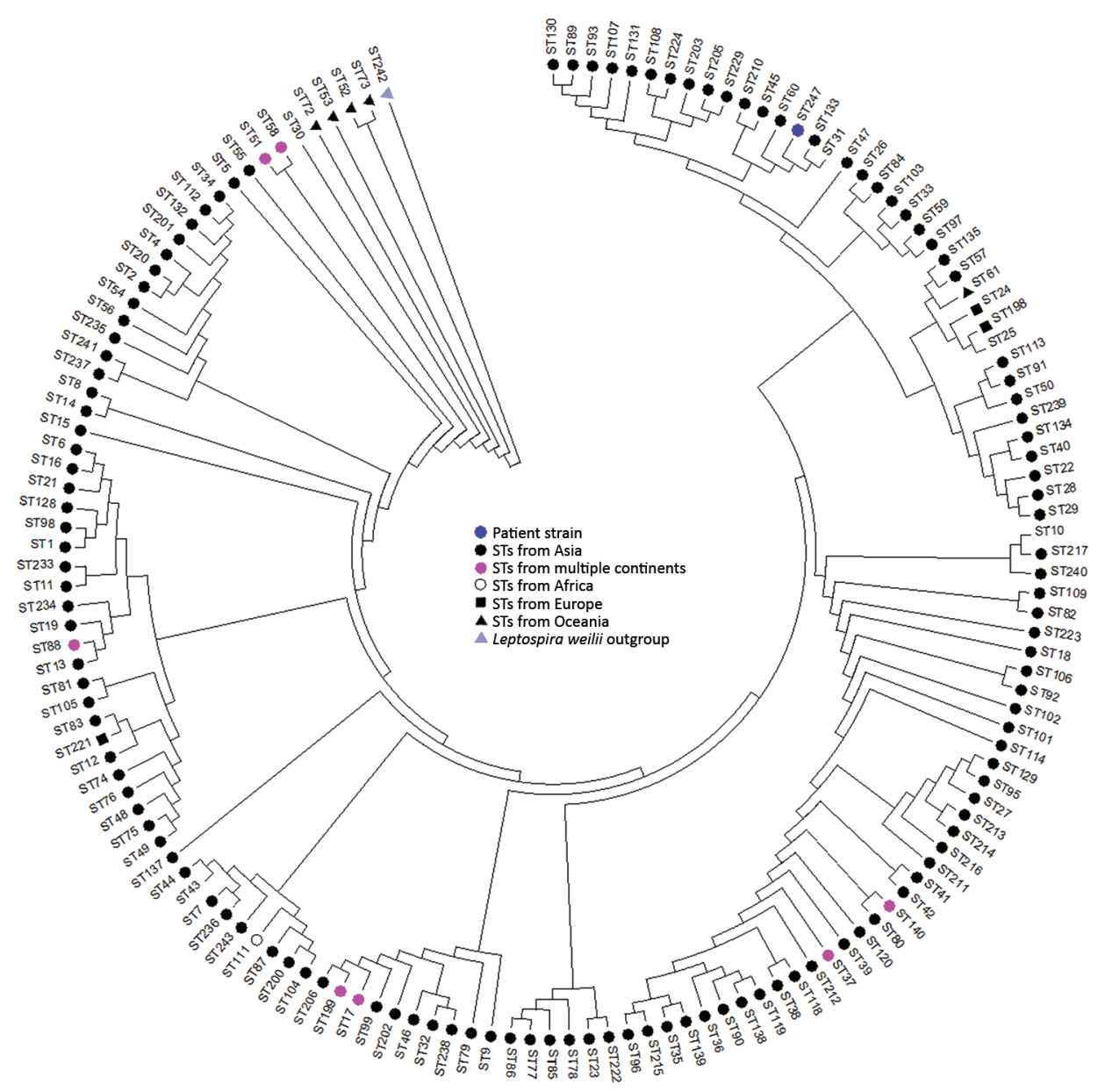

Figure 1. Phylogenetic tree showing only the topology for all Leptospira interrogans STs and their continent of origin. The isolate obtained from the patient in this study with severe myasthenic manifestation of leptospirosis is indicated by a blue dot (ST247). Tree was constructed using the maximum-likelihood method with MEGA version 7.0 (5). ST, sequence type.

No leptospiral DNA was found in the patient's cerebrospinal fluid; no Leptospira spp. IgG or IgM were detected by ELISA (Virion/Serion, https://www.serion-diagnostics. de). Several days later, a second serum sample showed high levels of $\operatorname{IgM}(>100 \mathrm{U} / \mathrm{mL})$.

The neurologic examination suggested MG, which was corroborated by pathologic decrements in compound muscle action potentials in the orbicularis oculi $(-15 \%)$ and trapezius $(-46 \%)$ muscles on repetitive nerve stimulation at $3 \mathrm{~Hz}$. Administration of the acetylcholinesterase inhibitor edrophonium resulted in strong and immediate improvement of ptosis, bulbar symptoms, and limb weakness. Nerve conduction studies revealed prolonged latency in the left medianus nerve, compatible with carpal tunnel syndrome. All other results were unremarkable, largely ruling out peripheral nerve or muscular disorders.

We consequently made a diagnosis of $\mathrm{MG}$ and acute leptospirosis and initiated treatment with pyridostigmine bromide and intravenous ceftriaxone. We withheld immunosuppressive therapy because of the acute leptospirosis. After 3 days, the patient demonstrated increased strength without further dysphagia or dysarthria. After 7 days, C-reactive protein levels normalized, proteinuria resolved, and gamma-glutamyltransferase levels decreased to $373 \mathrm{U} / \mathrm{L}$. Ceftriaxone was discontinued after 10 days. Repetitive nerve stimulation was repeated 1 week after the initial test without prior ingestion of pyridostigmine and showed improved function of the neuromuscular junction.

We did not detect antibodies against acetylcholine receptors (AChRs) and muscle-specific tyrosine kinase (MuSK). However, testing for antibodies against lowdensity lipoprotein receptor-related protein 4 (LRP4) gave weakly positive results in a cell-based indirect immunofluorescence assay in an independent external laboratory (Medizinisches Versorgungszentrum Labor Volkmann Karlsruhe, Karlsruhe, Germany).

Pyridostigmine was discontinued, and the patient was discharged symptom-free and in good health. At follow-up 1 month later, examination of the patient found only minor ocular weakness. Repetitive nerve stimulation of the orbicularis oculi and trapezius muscles yielded normal results. At 4- and 6-month follow-up visits, minor fatigability of 


\begin{tabular}{|l|lcccc|}
\hline \multirow{2}{*}{$\begin{array}{l}\text { Decrement in } \\
\text { repetitive stimulation }\end{array}$} & Trapezius & $-47 \%$ & $-3.6 \%$ & $-3.5 \%$ \\
\cline { 2 - 6 } & Orbicularis oculi & $-15 \%$ & $-13.3 \%$ & $-0.9 \%$ \\
\hline CRP, mg/dL & & 27.72 & 0.74 & 0.39 & 0.05 \\
\hline \multirow{3}{*}{ Serum antibodies } & Leptospira IgM & 12 & & $>100$ & 59 \\
\cline { 2 - 6 } & Leptospira IgG & 1 & 3 & 5 \\
\cline { 2 - 6 } & Anti-LRP4 & Not tested & Positive & \\
\hline
\end{tabular}

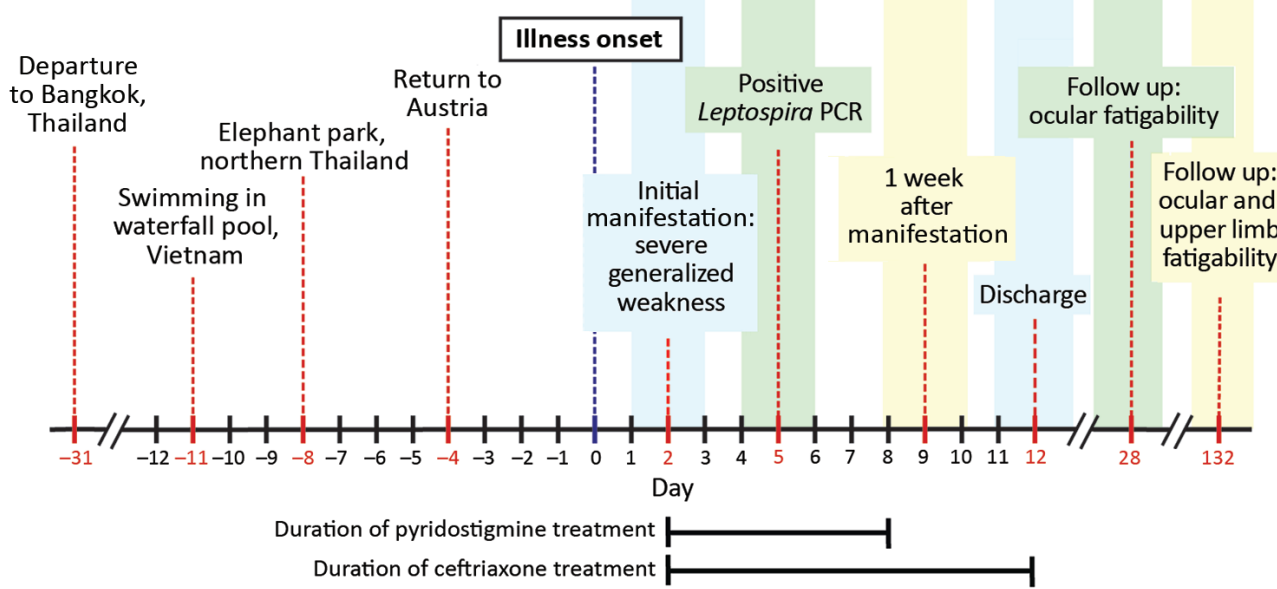

Figure 2. Timeline of medical history for patient with severe myasthenic manifestation of leptospirosis, including results of relevant neurologic and laboratory investigations. Decrement in repetitive stimulation denotes the maximum decrease in amplitude of the fourth or fifth compound muscle action potential waveform during supramaximal repetitive nerve stimulation at $3 \mathrm{~Hz}$. A decrement $>10 \%$ is regarded as pathologic (6). Leptospira ELISA cutoff values: IgG, $<10 \mathrm{U} / \mathrm{mL}$ negative, $15 \mathrm{U} /$ $\mathrm{mL}$ positive; IgM, $<15 \mathrm{U} / \mathrm{mL}$ negative, $>20 \mathrm{U} / \mathrm{mL}$ positive. CRP, C-reactive protein; LRP4, lipoprotein receptorrelated protein 4 . the lateral rectus muscle and the upper extremities was observed, but these effects did not impair the patient's performance in a physically demanding profession and did not require medication. The timeline of the patient's medical history is summarized in Figure 2.

MG is an acquired, antibody-mediated autoimmune disease of the neuromuscular junction causing muscle weakness and increased fatigability. Treatment consists of symptomatic relief with acetylcholinesterase inhibitors. Immunosuppression is often required for disease control. Approximately $85 \%$ of MG patients have AChR and MuSK antibodies (7); among the remaining patients, anti-LRP4 autoantibodies can be detected in $1.4 \%-50 \%$ of patients, depending on the detection method $(8,9)$. Cell-based assays with live cells appear associated with lower frequencies compared with fixed-cell assays or with ELISA. Samples from the patient we describe tested negative for AChR and MuSK antibodies, but testing against LRP4 antibodies gave positive results in an external laboratory and borderline positive results in an in-house live cell-based assay (10).

Our results should be interpreted cautiously. We were unable to reproduce them with remaining serum samples in the in-house live cell-based assay or ELISA at a later time.

Case series suggest an association of viral infections with the development of MG, although a causal link has yet to be shown $(11,12)$. Worsening of preexisting $\mathrm{MG}$ is often triggered by an infection. A registry study in Spain found life-threatening events related to MG in up to $10 \%$ of patients, with infection being the most common cause (13).
The patient we report had a myasthenic crisis associated with a new ST of $L$. interrogans. Only 1 other case of leptospirosis with involvement of the neuromuscular junction has been described (14), but without direct identification of the pathogen and with symptoms occurring after the leptospiremic phase in the presence of Leptospira-specific antibodies; no myasthenic antibodies were tested for, and no diagnosis of MG was made.

In contrast, the patient we describe experienced more severe weakness at a much earlier point, and his serum sample tested positive for LRP4 autoantibodies, suggesting an association between MG and the acute leptospirosis. Reports of leptospirosis-associated, immune-mediated manifestations ranging from mononeuritis and Guillain-Barrélike syndromes to postinfectious autoimmune epilepsy $(3,15)$ raise the question of whether Leptospira infections might precipitate or aggravate autoimmunity.

\section{Acknowledgments}

We thank Angela Vincent, David Beeson, and Lin Mei for use of their LRP4 plasmids.

I.K. was funded by a Hertha Firnberg Fellowship (project no. T996-B30) from the Austrian Science Fund.

\section{About the Authors}

Dr. Tomschik has been a resident in clinical neurology at the Medical University of Vienna since 2016 whose research currently focuses on neuromuscular diseases, particularly myasthenia gravis. Dr. Koneczny is a molecular biologist and project leader at the Medical University of Vienna whose research currently focuses on MG. 


\section{References}

1. Levett PN. Leptospirosis. Clin Microbiol Rev. 2001;14:296-326. http://dx.doi.org/10.1128/CMR.14.2.296-326.2001

2. Bharucha NE. Infections of the nervous system. In: Bradley's neurology in clinical practice. Bradley DR, Fenichel GM, editors. Butterworth Heinemann: Boston; 1991. p. 1074-5.

3. Panicker JN, Mammachan R, Jayakumar RV. Primary neuroleptospirosis. Postgrad Med J. 2001;77:589-90. http://dx.doi.org/10.1136/pmj.77.911.589

4. Boonsilp S, Thaipadungpanit J, Amornchai P, Wuthiekanun V, Bailey MS, Holden MT, et al. A single multilocus sequence typing (MLST) scheme for seven pathogenic Leptospira species. PLoS Negl Trop Dis. 2013;7:e1954. http://dx.doi.org/10.1371/ journal.pntd.0001954

5. Kumar S, Stecher G, Tamura K. MEGA7: Molecular Evolutionary Genetics Analysis version 7.0 for bigger datasets. Mol Biol Evol. 2016;33:1870-4. http://dx.doi.org/10.1093/molbev/msw054

6. AAEM Quality Assurance Committee. American Association of Electrodiagnostic Medicine. Literature review of the usefulness of repetitive nerve stimulation and single fiber EMG in the electrodiagnostic evaluation of patients with suspected myasthenia gravis or Lambert-Eaton myasthenic syndrome. Muscle Nerve. 2001;24:1239-47. http://dx.doi.org/ $10.1002 /$ mus. 1140

7. Vincent A, Huda S, Cao M, Cetin H, Koneczny I, Rodriguez-Cruz P, et al. Serological and experimental studies in different forms of myasthenia gravis. Ann N Y Acad Sci. 2018;1413:143-53. http://dx.doi.org/10.1111/nyas.13592

8. Yan M, Xing GL, Xiong WC, Mei L. Agrin and LRP4 antibodies as new biomarkers of myasthenia gravis. Ann N Y Acad Sci. 2018;1413:126-35. http://dx.doi.org/10.1111/nyas.13573

9. Zisimopoulou P, Evangelakou P, Tzartos J, Lazaridis K, Zouvelou V, Mantegazza R, et al. A comprehensive analysis of the epidemiology and clinical characteristics of anti-LRP4 in myasthenia gravis. J Autoimmun. 2014;52:139-45. http://dx.doi.org/10.1016/j.jaut.2013.12.004

10. Rodríguez Cruz PM, Al-Hajjar M, Huda S, Jacobson L, Woodhall M, Jayawant S, et al. Clinical features and diagnostic usefulness of antibodies to clustered acetylcholine receptors in the diagnosis of seronegative myasthenia gravis. JAMA Neurol. 2015;72:642-9. http://dx.doi.org/10.1001/jamaneurol.2015.0203

11. Leis AA, Szatmary G, Ross MA, Stokic DS. West Nile virus infection and myasthenia gravis. Muscle Nerve. 2014;49:26-9 http://dx.doi.org/10.1002/mus.23869

12. Molko N, Simon O, Guyon D, Biron A, Dupont-Rouzeyrol M, Gourinat AC. Zika virus infection and myasthenia gravis: report of 2 cases. Neurology. 2017;88:1097-8. http://dx.doi.org/10.1212/ WNL.0000000000003697

13. Ramos-Fransi A, Rojas-García R, Segovia S, Márquez-Infante C, Pardo J, Coll-Cantí J, et al.; Myasthenia NMD-ES Study Group. Myasthenia gravis: descriptive analysis of life-threatening events in a recent nationwide registry. Eur J Neurol. 2015;22:1056-61. http://dx.doi.org/10.1111/ene.12703

14. Pradhan S, Tandon R, Kishore J. Combined involvement of muscle, nerve, and myoneural junction following Leptospira infection. Neurol India. 2012;60:514-6. http://dx.doi.org/10.4103/ 0028-3886.103202

15. Makhija P, Gopinath S, Kannoth S, Radhakrishnan K. A case of post-leptospirosis autoimmune epilepsy presenting with sleep-related hypermotor seizures. Epileptic Disord. 2017; $19: 456-60$

Address for correspondence: Matthias Tomschik, Medical University of Vienna, Department of Neurology, Spitalgasse 23, 1090 Vienna, Austria; email: matthias.tomschik@meduniwien.ac.at

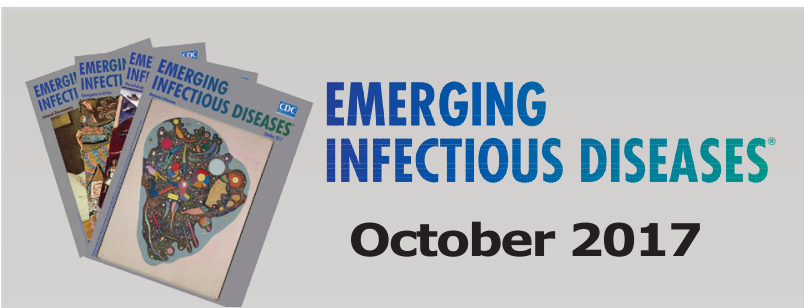

\section{Bacterial Infections}

- Fatal Rocky Mountain Spotted Fever along the United States-Mexico Border, 2013-2016

- Surveillance of Extrapulmonary Nontuberculous Mycobacteria Infections, Oregon, USA, 2007-2012

- Investigation of Outbreaks of Salmonella enterica Serovar Typhimurium and Its Monophasic Variants Using WholeGenome Sequencing, Denmark

- Enteric Infections Circulating during Haii Seasons, 2011-2013

- Economic Assessment of Waterborne Outbreak of Cryptosporidiosis

- Antimicrobial Drug Prescription and Neisseria gonorrhoeae Susceptibility, United States, 2005-2013

- Poliovirus Excretion in Children with Primary Immunodeficiency Disorders, India

- Disease Burden of Clostridium difficile Infections in Adults, Hong Kong, China, 2006-2014

- Molecular Tracing to Find Source of Protracted Invasive Listeriosis Outbreak, Southern Germany, 2012-2016

- Dengue Virus 1 Outbreak in Buenos Aires, Argentina, 2016

- Mild Illness during Outbreak of Shiga Toxin-Producing Escherichia coli O157 Infections Associated with Agricultural Show, Australia

- Enterovirus D68-Associated Acute Flaccid Myelitis in Immunocompromised Woman, Italy

- Diagnosis of Fatal Human Case of St. Louis Encephalitis Virus Infection by Metagenomic Sequencing,

California, 2016

- Usutu Virus RNA in Mosquitoes, Israel, 2014-2015

- Macrolide-Resistant Mycoplasma pneumoniae Infection, Japan, 2008-2015

- Epidemiology of Reemerging Scarlet Fever, Hong Kong, 2005-2015

- Off-Label Use of Bedaquiline in Children and Adolescents with Multidrug-Resistant Tuberculosis

- Monitoring Avian Influenza Viruses from Chicken Carcasses Sold at Markets, China, 2016

- Berlin Squirrelpox Virus, a New Poxvirus in Red Squirrels, Berlin, Germany

\section{To revisit the October 2017 issue, go to: https://wwwnc.cdc.gov/eid/articles/ issue/23/10/table-of-contents}

